\title{
Is Citrullination the Missing Link between Periodontal Disease and Rheumatoid Arthritis?
}

\author{
Anna B. Montgomery • Elena B. Lugli • \\ Patrick J. Venables
}

Published online: 6 January 2015

(C) Springer International Publishing AG 2015

\begin{abstract}
Connections between periodontitis (PD) and rheumatoid arthritis (RA) are suggested by an increased prevalence of PD in RA, shared environmental and genetic risk factors, and correlating levels of severity when the two diseases occur together. Here, we compare and contrast the results from numerous studies documenting this association, and highlight the involvement of citrullination in the development of autoimmunity leading to the destructive pathology that characterizes RA. The contribution of citrullination to RA may occur in at least three distinct phases: (i) the initial breakdown of tolerance leading to autoimmunity; (ii) the maturation of the citrulline specificity of the autoantibody response; and (iii) the pro-inflammatory effect of citrullinated proteins themselves in established disease. We conclude that citrullination is more than a 'missing link'; rather, it is an active process in the evolution of low levels of autoimmunity found in PD into the pathogenic anti-citrullinated protein response specific to RA.
\end{abstract}

Keywords Rheumatoid arthritis · Periodontitis . Citrullination - Anti-citrullinated protein antibodies (ACPA) . Peptidylarginine deiminase (PAD) · Etiology ·

Porphyromonas gingivalis $\cdot$ PPAD

This article is part of the Topical Collection on Systemic Diseases

A. B. Montgomery · E. B. Lugli • P. J. Venables $(\bowtie)$

Kennedy Institute of Rheumatology, University of Oxford,

Roosevelt Drive, Headington, Oxford OX3 7FY, UK

e-mail: patrick.venables@kennedy.ox.ac.uk

A. B. Montgomery

e-mail: anna.montgomery@kennedy.ox.ac.uk

E. B. Lugli

e-mail: elena.lugli@kennedy.ox.ac.uk

\section{Introduction}

Rheumatoid arthritis (RA) is a chronic inflammatory disease characterized by inflammation and destruction of the joints. The etiology of RA is unknown, but increasing evidence suggests it fulfills the paradigm of the interaction between genetic and environmental factors leading to a disease-specific autoimmune response [1]. Periodontitis (PD) is one candidate environmental risk factor. In this review we consider the main elements of the interaction between RA and PD, and examine citrullination as the process linking them.

\section{Citrullination in Health and Disease}

The post-translation modification of citrullination is the conversion of an arginine residue to a citrulline residue by peptidylarginine deiminase (PAD) enzymes, and occurs constitutively in humans by five known PAD isoforms (PAD1-4 and PAD6). With broad tissue distribution, PADs carry out citrullination in a wide range of processes, from keratinization of skin to innate immunity [2]. Of particular interest in RA and PD are isoforms PAD2 and PAD4 as both have been found in the RA synovium [3] and in periodontal tissues in PD [4]. PAD2 is found in multiple tissues and skeletal muscle, while PAD4 is found predominantly in macrophages and neutrophils [2]. Deregulated levels of citrullination have been implicated in a number of pathological conditions including psoriasis and prion disease [5, 6]. A well-characterized example is multiple sclerosis (MS), a neurodegenerative disease characterized by the destruction of myelin around nerve axons. In MS, citrullination of myelin basic protein in brain tissues is increased, altering the myelin structure, increasing susceptibility to degradation by proteases, and potentially exposing neoepitopes for responding T cells [7]. Citrullination is also a feature of a number of inflammatory diseases, including both 
RA and PD, but antibodies to citrullinated protein/peptide antigens (anti-citrullinated protein antibodies; ACPA) remain relatively specific for RA.

\section{The Chain of Events Between Periodontitis and Rheumatoid Arthritis}

An association between PD and RA has been theorized since Hippocrates described pulling teeth as a cure for arthritis circa 400 BC. In more recent years the link between the two diseases has been substantiated by several studies based on different populations from several continents. Despite the definition of periodontal disease status and RA manifestations often differing between studies, the vast majority reported a positive association, with increased prevalence of PD in patients with RA compared to osteoarthritis (OA) or controls without joint disease (Table 1). The association between the two diseases appeared to be independent of smoking status in several studies [8-12], as did other parameters and confounders such as age, gender, and medication $[10,11$, 13-15]. However, it is important to note that these parameters do vary between studies and, in particular, the use of antiinflammatory drugs such as corticosteroids, non-steroidal anti-inflammatory drugs, and disease-modifying anti-rheumatic drugs can mask true periodontal conditions, especially when evaluating less severe periodontal conditions $[16,17]$ (reviewed in [18]). The influence of oral microbes normally associated with PD and their effect on RA has also been looked at in some studies $[8,9,15,19 \cdot, 20]$. The presence of, and antibodies to, Porphyromonas gingivalis, one of the main bacterial species implicated in the etiology and pathogenesis of PD, often correlates with RA parameters. One study found an association between new-onset RA and bacterial species other than $P$. gingivalis (Prevotella and Leptotrichia) irrespective of severity of PD. These bacteria were not present in the control group. Interestingly, the genus Porphyromonas was universally present in all patient groups but $P$. gingivalis specifically was the most prevalent in PD patients. The study also showed that in the newonset RA group, $P$. gingivalis was more prevalent and abundant in patients with advanced PD than in those without PD [8].

A number of studies have observed a correlation in the severity of the conditions: de Smit et al. [9] found RA patients with severe PD had a higher 28-joint Disease Activity Score (DAS28) than RA patients with no or moderate PD. Similarly Mercado et al. found periodontal bone loss correlated to swollen joint count and health assessment status in RA [21]. In a large and comprehensive study, Gonzalez et al. [20] also measured loss of periodontal bone in relation to a variety of ACPA specificities in 287 RA patients and 330 OA controls. They observed significantly increased bone loss in ACPA- positive patients, which correlated with total ACPA levels and health assessment questionnaire, tender joint count and DAS28-CRP (C-reactive protein) scores. Interestingly, of 19 reactivities tested, levels of anti-citrullinated vimentin and anti-citrullinated histone antibodies also correlated with increased bone loss [20]. In another study on this cohort, Mikuls et al. [19•] investigated ACPA level and specificity in relation to $\mathrm{PD}$ status and antibodies to $P$. gingivalis surface proteins. They found a correlation between anti$P$. gingivalis antibodies and total ACPA level, and a correlation between anti-P. gingivalis antibodies and ACPA specificity, which appeared to be dependent on smoking status. Anti-citrullinated-clusterin, enolase, filaggrin, and fibrinogen antibodies were associated with anti- $P$. gingivalis levels in the 'ever-smokers' group, while 'never smokers' had correlating anti- $P$. gingivalis and citrullinated-filaggrin, histone, and vimentin antibodies [19॰]. These studies demonstrate a strong correlation between PD and RA severity, which is associated with ACPA production. There also appears to involvement of $P$. gingivalis, which is modulated by smoking status, demonstrating the importance of environmental factors in disease pathogenesis.

\section{Interaction with Genetics}

The most well-established genetic risk factor for RA is the 'shared epitope' (SE), a number of $H L A-D R B 1$ alleles encoding five amino acids around the binding cleft of the major histocompatibility complex (MHC) class II molecule. These include members of the $H L A-D R B 1^{*} 04$ group, $H L A$ $D R B 1^{*} 0101$ and $* 0102$, as well as lesser-known alleles (reviewed by Holoshitz [22]). These particular amino acid arrangements influence binding of peptides for antigen presentation, and generally exhibit higher affinity for negative or uncharged amino acids over positive, thus making binding of neutral citrulline preferential to positively charged arginine [23]. SE alleles are also associated with chronic aggressive PD [24], though it remains unclear whether the SE is associated with PD as a whole. The PTPN22 R620W gene variant is another known susceptibility factor in RA [25] and with a stronger association with ACPA-positive RA [26]. However, the role of this variant of PTPN22 in relation to PD remains to be established.

\section{Interactions with Smoking}

Cigarette smoking is an established environmental risk factor for RA, and has a stronger association with the ACPA-positive phenotype [27]. It has been suggested that this may be due to increased expression of PAD enzymes in the lungs of smokers [28], leading to citrullination of native proteins in the lung, 
Table 1 Prevalence and severity of periodontitis in rheumatoid arthritis in various studies

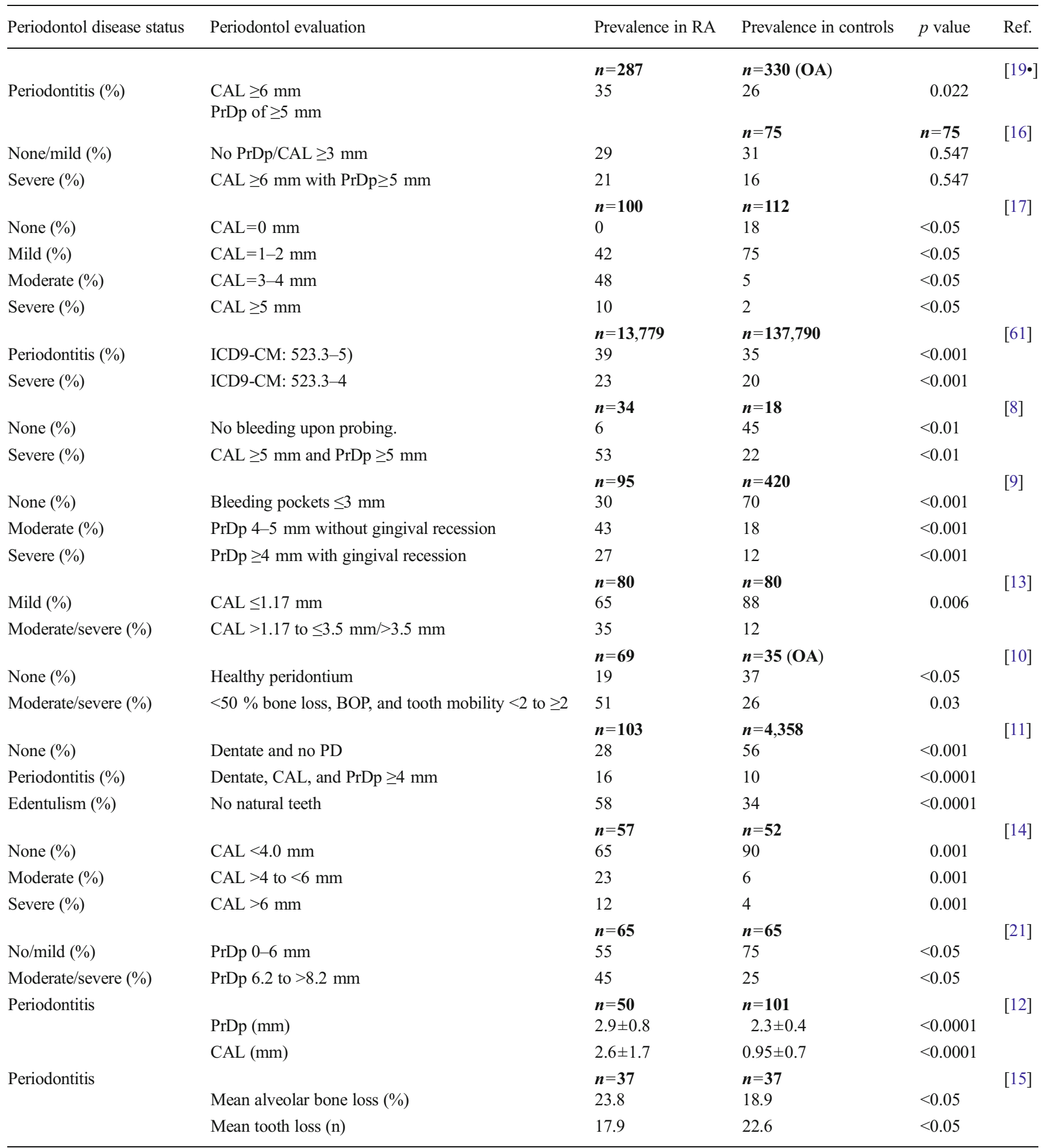

$C A L$ clinical attachment loss, ICD9-CM International Classification of Disease, 9th revision, clinical modification, $O A$ osteoarthritis, $\operatorname{Pr} D p$ probing depths

which in turn results in the formation of ACPA. Smoking is also a risk factor for PD and tooth loss in a dose-dependent manner [29, 30], but it is now accepted that the links between $\mathrm{PD}$ and RA cannot be explained by smoking as a common risk factor [8-10].

\section{Anti-Citrullinated Protein Antibodies: the Link in the Chain}

The discovery of ACPA has provided the biggest step in recent years towards refining the diagnosis of RA [31] and increasing 
our understanding of its etiopathogenesis (reviewed in Wegner et al. [1]). Diagnostic tests for ACPA commonly use cyclic synthetic peptides such as the anti-cyclic citrullinated peptide (anti-CCP) assays, or mutated forms of recombinant proteins citrullinated in vitro such as the anti-mutated citrullinated vimentin assay. These generic tests for ACPA are now included in the revised diagnostic criteria for RA [32] and are associated with more aggressive disease measured by DAS28, Visual Analogue Scale, and radiographic scores of joint damage [33-35] as well as an increased risk of secondary disorders of the cardiovascular system [36]. ACPA are present in serum years before disease onset $[37,38]$ and increase in titre and range of specificities prior to development of pathology [34, 39]. A recent study reported reduced bone volume and bone mineral density loss in a small group of ACPA-positive individuals with no arthralgia or joint swelling [40], suggesting that ACPA may induce bone loss even before clinical presentation of symptoms.

In addition to clinical correlations of ACPA with disease severity, there is also evidence of direct involvement of ACPA in pathogenesis. The ACPA tests, which are so valuable in diagnosis, are of limited use when it comes to examining pathogenic mechanisms. This is because the peptides used in the CCP assays, for example, were selected on the basis of their ability to maximize the diagnostic sensitivity of the antibody tests, and do not represent the real antigens that occur in vivo. Therefore, the so-called 'specific antigens' are those that provide the best tools for examining the pathogenicity of ACPA. The best-established specific antigens are the citrullinated forms of fibrinogen, vimentin, enolase, and type-2 collagen (reviewed in Wegner et al. [1]). Harre et al. demonstrated that anti-citrullinated vimentin antibodies stimulate osteoclastogenesis by binding citrullinated vimentin on the cell surface [41]. In addition, binding of immune complexes containing antibody and citrullinated fibrinogen induced increased production of tumor necrosis factor- $\alpha$ by simultaneously binding both toll-like receptor 4 (TLR4) and the Fc-gamma receptor [42•]. In this study, Sokolove and colleagues also demonstrated that citrullinated fibrinogen alone induced a more potent cytokine response than native fibrinogen through TLR4 and MyD88 pathway activation, indicating citrullinated proteins themselves may be pathogenic.

\section{Citrullination: the Missing Link?}

In light of increasing evidence that ACPA are directly involved in RA disease pathogenesis, it is reasonable to propose that citrullination of autoantigens is an essential process that forms a link (or links) in the chain of events between etiological agents and the downstream events in disease pathology. PD is particularly relevant in this respect, because one of its major pathogens, $P$. gingivalis, expresses the only known bacterial PAD enzyme known as $P$. gingivalis PAD (PPAD). PPAD has attracted considerable attention as a possible molecule underlying the mechanisms for tolerance breakdown in RA. It differs from mammalian PADs in its ability to citrullinate free arginine and also shows a marked preference for C-terminal peptidyl arginines generated by another $P$. gingivalis enzyme, arginine gingipain, whereas eukaryotic PADs only citrullinate internal arginines. PPAD also differs in its lack of $\mathrm{Ca}^{2+}$ dependency and therefore does not depend on cell lysis, apoptosis, or secretion for its activity. The citrullinating capabilities of PPAD are thought to promote bacterial survival of $P$. gingivalis by inactivating the killing defenses of the host, such as antimicrobial peptides and complement $[43,44]$. In vitro PPAD citrullinates peptides with C-terminal arginines from RA-associated antigens, enolase and fibrinogen [45]. Given C-terminally citrullinated peptides would not be generated by host PAD enzymes such as PAD2 and PAD4, these would be true neopeitopes and thus could potentially react with $\mathrm{T}$ cells to drive an antibody response against both bacterially derived and host proteins. One problem with this hypothesis is the lack of evidence that C-terminally citrullinated peptides bind to $\mathrm{MCH}$ molecules or to responding $\mathrm{T}$ cell receptors. However, one group has shown that a highly immunogenic peptide from hen egg lysozyme (HEL) was citrullinated by endogenous PADs in a C-subterminal position (flanked only by a downstream Cterminal glycine) and bound to MHC. This interaction resulted in autoantibodies to uncitrullinated HEL and autophagy in HEL transgenic mice [46, 47]. Thus, it remains possible that C-terminally citrullinated peptides from enolase or fibrinogen may be bound to MHC and break tolerance by similar mechanisms. In support of this idea, is the observation that patients with PD have antibodies to peptides from enolase and other RA autoantigens and that these antibodies, as in the HEL transgenic mice, reacted mainly with uncitrullinated variants of the peptides [48, $49 \cdot \bullet$.

A second mechanism for PPAD breaking tolerance to citrullinated proteins is via autocitrullination, which occurs during the production and purification of recombinant PPAD [50]. Citrullinated peptides from PPAD are targeted by antibodies in RA [50, 51] but autocitrullination of PPAD has yet to be demonstrated in vivo, so it is possible that the antibodies to citrullinated PPAD peptides are simply part of the crossreactive ACPA response. However, if autocitrullination of PPAD does prove to be a mechanism for inducing autoimmunity in RA, it has profound therapeutic implications, in that PPAD inhibitors could be a novel and targeted approach to treating patients whose RA has been induced by PD. Since PPAD is structurally and genetically unrelated to mammalian PADs, such treatment should be antibacterial and therefore devoid of side effects. 
A third mechanism for citrullination breaking tolerance may not involve PPAD at all but the host PADs, such as PAD2 and PAD4. A polymorphism in the PADI4 gene, which results in increased stability of PAD4 mRNA, has been shown to be a susceptibility factor in RA in Asian populations [52]. This suggests that increased citrullination by PAD4 could be directly linked to ACPA formation, possibly by generating neoepitopes that bind favorably to the MHC. One of the first studies to examine this mechanism showed that a peptide derived from the autoantigen vimentin (amino acids 66-77), with arginine-71 replaced by a citrulline, binds with high affinity to HLA-DRB $1 * 0401$ MHC class II molecules, eliciting T cell activation [23].

In PD, citrullinated proteins and enzymes PAD2 and PAD4 have been detected in gingival tissues [4], and have been shown in higher levels in inflamed tissues, correlating with clinical parameters of pathology such as depth of periodontal pockets [53]. Both PAD2 and PAD4 are important in the formation of neutrophil extracellular traps (NETs), which have been detected in abundance in purulent crevicular fluid from patients with PD [54]. It has been proposed by Dwivedi and Radic that NET formation may induce autoimmunity to citrullinated proteins in RA through internalization of bacteria complexed with citrullinated proteins, inducing an antibody response to both bacterial and native proteins [55]. Expression of key citrullinated RA antigens vimentin and enolase on the surface of NETs demonstrated by Sur Chowdhury et al., [56] supports this hypothesis. In addition, an antibody response to the citrullinated histones mediating NET formation has been observed in RA patients [57].

A fourth possible mechanism for inducing ACPA may not involve citrullination at all, at least not in the initial stages. Of relevance in this respect is the immunodominant epitope of citrullinated alpha enolase known as citrullinated enolase peptide 1 (CEP-1). The sequence KIHA-cit-EIFDScit-GNPTVE is $97 \%$ homologous to the corresponding sequence in citrullinated $P$. gingivalis enolase [58]. Thus, $P$. gingivalis infection could initiate tolerance breakdown through cross-reactivity of antibacterial enolase immunity with mammalian enolase. This was demonstrated in an animal model by Kinloch et al., where immunization of DR4 transgenic mice with both citrullinated and uncitrullinated $P$. gingivalis enolase induced autoantibodies to mammalian enolase. Importantly, the antibody response was not citrulline specific as judged by reactivity of serum from the immunized mice with the arginine control peptide (REP-1) [59]. This indicates that citrullination is not required for inducing an immune response, and that when the response occurs, the antibodies are not citrulline specific. This is in keeping with the findings of largely not citrulline-specific autoantibody response to CEP-1 and REP-1 in PD [48, 49••] and a very similar pattern of non-citrulline-specific ACPA responses in another chronic bacterial infection, bronchiectasis [60]. At first sight, the lack of citrulline specificity in these immune responses would seem to conflict with the numerous studies demonstrating ACPA in the serum of RA patients years before the onset of disease. However, one very recent reanalysis of a large pre-RA study showed that the earliest serum samples had a significantly higher response to the arginine control peptides from several citrullinated peptide antigens [59].

\section{Conclusion}

The links between PD and RA are now well-established in a number of epidemiological studies, both in terms of frequency and the severity of the two diseases. The evolution of pathogenic antibodies to citrullinated proteins is integral to this chain of events, suggesting that citrullination of autoantigens must occur as part of the process. Citrullination could promote tolerance breakdown, but the evidence to date suggests that it is likely to be at the T cell level, since the $\mathrm{B}$ cell response in diseases that predispose to RA (including PD) is not citrulline specific. By contrast, by the time the first signs of arthritis appear, the ACPA response is already strikingly citrulline specific, suggesting that constant exposure to citrullinated autoantigens at the site of inflammation, be it in the periodontium, lungs or possibly even the gastrointestinal tract, causes a second link, the gradual evolution of citrulline specificity of the ACPA response, which further matures as a result of exposure to citrullinated proteins in the joint. Once clinical disease becomes established, citrullination of autoantigens may acquire a third pathogenic role by interacting with TLR receptors, already well-characterized in the cases of fibrinogen and vimentin, with other proinflammatory citrullinated proteins awaiting discovery.

Thus, we can conclude the citrullination is more than a 'missing link', it is an essential process in the evolution of autoimmunity in those patients with PD who go on to develop RA. This is important because inhibition of the bacterial enzyme PPAD, or perhaps the host PADs, PAD2 and PAD4, could be a completely novel approach to treating RA by specifically targeting this link.

\section{Compliance with Ethics Guidelines}

Conflict of Interest Anna B. Montgomery, Elena B. Lugli, and Patrick J. Venables declare that they have no conflict of interest.

Human and Animal Rights and Informed Consent Both human and animal studies, all with appropriate ethical approval, informed consent and observation of national guidelines for animal welfare. 


\section{References}

Papers of particular interest, published recently, have been highlighted as:

- Of importance

•- Of major importance

1. Wegner N, Lundberg K, Kinloch A, et al. Autoimmunity to specific citrullinated proteins gives the first clues to the etiology of rheumatoid arthritis. Immunol Rev. 2010;233(1):34-54.

2. Wang S, Wang Y. Peptidylarginine deiminases in citrullination, gene regulation, health and pathogenesis. Biochim Biophys Acta. 2013;1829(10):1126-35.

3. Kinloch A, Lundberg K, Wait R, et al. Synovial fluid is a site of citrullination of autoantigens in inflammatory arthritis. Arthritis Rheum. 2008;58(8):2287-95.

4. Nesse W, Westra J, van der Wal JE, et al. The periodontium of periodontitis patients contains citrullinated proteins which may play a role in ACPA (anti-citrullinated protein antibody) formation. J Clin Periodontol. 2012;39(7):599-607.

5. Ishida-Yamamoto A, Senshu T, Takahashi H, et al. Decreased deiminated keratin $\mathrm{K} 1$ in psoriatic hyperproliferative epidermis. J Invest Dermatol. 2000;114(4):701-5.

6. Jang B, Ishigami A, Maruyama N, et al. Peptidylarginine deiminase and protein citrullination in prion diseases: strong evidence of neurodegeneration. Prion. 2013;7(1):42-6.

7. Moscarello MA, Lei H, Mastronardi FG, et al. Inhibition of peptidyl-arginine deiminases reverses protein-hypercitrullination and disease in mouse models of multiple sclerosis. Dis Model Mech. 2013;6(2):467-78.

8. Scher JU, Ubeda C, Equinda M, et al. Periodontal disease and the oral microbiota in new-onset rheumatoid arthritis. Arthritis Rheum. 2012;64(10):3083-94.

9. de Smit M, Westra J, Vissink A, et al. Periodontitis in established rheumatoid arthritis patients: a cross-sectional clinical, microbiological and serological study. Arthritis Research \& Therapy 2012: 14:R222.

10. Dissick A, Redman RS, Jones M, et al. Association of periodontitis with rheumatoid arthritis: a pilot study. J Periodontol. 2010;81(2): 223-30.

11. de Pablo P, Dietrich T, McAlindon TE. Association of periodontal disease and tooth loss with rheumatoid arthritis in the US population. J Rheumatol. 2008;35(1):70-6.

12. Kasser UR, Gleissner C, Dehne F, et al. Risk for periodontal disease in patients with longstanding rheumatoid arthritis. Arthritis Rheum. 1997;40(12):2248-51.

13. Abdelsalam SK, Hashim NT, Elsalamabi EM, et al. Periodontal status of rheumatoid arthritis patients in khartoum state. BMC Res Notes. 2011:4:460.

14. Pischon N, Pischon T, Kröger J, et al. Association among rheumatoid arthritis, oral hygiene, and periodontitis. J Periodontol. 2008;79 (6):979-86.

15. Tolo K, Jorkjend L. Serum antibodies and loss of periodontal bone in patients with rheumatoid arthritis. J Clin Periodontol. 1990;17(5): 288-91.

16. Susanto H, Nesse W, Kertia N, et al. Prevalence and severity of periodontitis in Indonesian patients with rheumatoid arthritis. J Periodontol. 2013;84(8):1067-74.

17. Rosamma J, Rajappan S, Nath SG, et al. Association between chronic periodontitis and rheumatoid arthritis: a hospital-based case-control study. Rheumatol Int. 2013;33(1):103-9.

18. Rutger Persson, G., Rheumatoid arthritis and periodontitis - inflammatory and infectious connections. Review of the literature. J Oral Microbiol, 2012;4.
19. Mikuls TR, Payne JB, Yu F, et al. Periodontitis and Porphyromonas gingivalis in patients with rheumatoid arthritis. Arthritis Rheumatol 2014;66(5):1090-100. An up-to-date, well-controlled study documenting the links between perionditis, Porphyromonas gingivalis infection and seropositive rheumatoid arthritis.

20. Gonzalez, S.M., Payne JB, Yu F, et al., Alveolar bone loss is associated with circulating anti-citrullinated protein antibody (ACPA) in rheumatoid arthritis patients. J Periodontol, 2014: p. 1-16.

21. Mercado FB, Marshall RI, Klestov AC, et al. Relationship between rheumatoid arthritis and periodontitis. J Periodontol. 2001;72(6): 779-87.

22. Holoshitz J. The rheumatoid arthritis HLA-DRB1 shared epitope. Curr Opin Rheumatol. 2010;22(3):293-8.

23. Hill JA, Southwood S, Sette A, et al. Cutting edge: the conversion of arginine to citrulline allows for a high-affinity peptide interaction with the rheumatoid arthritis-associated HLA-DRB1*0401 MHC class II molecule. J Immunol. 2003;171(2):538-41.

24. Bonfil JJ, Dillier FL, Mercier P, et al. A "case control" study on the role of HLA DR4 in severe periodontitis and rapidly progressive periodontitis. Identification of types and subtypes using molecular biology (PCR.SSO). J Clin Periodontol. 1999;26(2):77-84.

25. Menard L, Saadoun D, Isnardi I, et al. The PTPN22 allele encoding an R620W variant interferes with the removal of developing autoreactive B cells in humans. J Clin Invest. 2011;121(9): 3635-44.

26. Feitsma AL, Toes RE, Begovich AB, et al. Risk of progression from undifferentiated arthritis to rheumatoid arthritis: the effect of the PTPN22 1858 T-allele in anti-citrullinated peptide antibody positive patients. Rheumatology (Oxford). 2007;46(7):1092-5.

27. Klareskog L, Malmström V, Lundberg K, et al. Smoking, citrullination and genetic variability in the immunopathogenesis of rheumatoid arthritis. Semin Immunol. 2011;23(2):92-8.

28. Makrygiannakis D, Hermansson M, Ulfgren AK, et al. Smoking increases peptidylarginine deiminase 2 enzyme expression in human lungs and increases citrullination in BAL cells. Ann Rheum Dis. 2008;67(10):1488-92.

29. Albandar JM, Streckfus CF, Adesanya MR, et al. Cigar, pipe, and cigarette smoking as risk factors for periodontal disease and tooth loss. J Periodontol. 2000;71(12):1874-81.

30. Genco RJ, Borgnakke WS. Risk factors for periodontal disease. Periodontol 2000. 2013;62(1):59-94.

31. Schellekens GA, de Jong BA, van den Hoogen FH, et al. Citrulline is an essential constituent of antigenic determinants recognized by rheumatoid arthritis-specific autoantibodies. J Clin Invest. 1998;101(1): 273-81.

32. Aletaha D, Neogi T, Silman AJ, et al. 2010 rheumatoid arthritis classification criteria: an American College of Rheumatology/ European League Against Rheumatism collaborative initiative. Ann Rheum Dis. 2010;69(9):1580-8.

33. Fisher BA, Plant D, Brode M, et al. Antibodies to citrullinated alpha-enolase peptide 1 and clinical and radiological outcomes in rheumatoid arthritis. Ann Rheum Dis. 2011;70(6):1095-8.

34. van der Woude D, Syversen SW, van der Voort EI, et al. The ACPA isotype profile reflects long-term radiographic progression in rheumatoid arthritis. Ann Rheum Dis. 2010;69(6):1110-6.

35. van de Stadt LA, van der Horst AR, de Koning MH, et al. The extent of the anti-citrullinated protein antibody repertoire is associated with arthritis development in patients with seropositive arthralgia. Ann Rheum Dis. 2011;70(1):128-33.

36. Aubart F, Crestani B, Nicaise-Roland P, et al. High levels of anticyclic citrullinated peptide autoantibodies are associated with cooccurrence of pulmonary diseases with rheumatoid arthritis. J Rheumatol. 2011;38(6):979-82.

37. Rantapää-Dahlqvist S, de Jong BA, Berglin E, et al. Antibodies against cyclic citrullinated peptide and $\operatorname{IgA}$ rheumatoid factor 
predict the development of rheumatoid arthritis. Arthritis Rheum. 2003;48(10):2741-9.

38. van de Stadt LA, de Koning MH, van de Stadt RJ, et al. Development of the anti-citrullinated protein antibody repertoire prior to the onset of rheumatoid arthritis. Arthritis Rheum. 2011;63 (11):3226-33.

39. Sokolove J, Bromberg R, Deane KD, et al. Autoantibody epitope spreading in the pre-clinical phase predicts progression to rheumatoid arthritis. PLoS One. 2012;7(5):e35296.

40. Kleyer A, Finzel S, Rech J, et al. Bone loss before the clinical onset of rheumatoid arthritis in subjects with anticitrullinated protein antibodies. Ann Rheum Dis. 2014;73(5):854-60.

41. Harre U, Georgess D, Bang H, et al. Induction of osteoclastogenesis and bone loss by human autoantibodies against citrullinated vimentin. J Clin Invest. 2012;122(5):1791-802.

42. Sokolove J, Zhao X, Chandra PE, et al. Immune complexes containing citrullinated fibrinogen costimulate macrophages via Toll-like receptor 4 and Fcgamma receptor. Arthritis Rheum. 2011;63(1):53-62. This study decribes two major pathogenic mechanisms whereby immune complexes containg autantibodies and their citrullinated antigens mediate inflammation and tissue destruction in the rheumatoid joint.

43. Koziel J, Bryzek D, Sroka A, et al. Citrullination alters immunomodulatory function of LL-37 essential for prevention of endotoxin-induced sepsis. J Immunol. 2014;192(11):5363-72.

44. Bielecka E, Scavenius C, Kantyka T, et al. Peptidyl arginine deiminase from Porphyromonas gingivalis abolishes anaphylatoxin C5a activity. J Biol Chem. 2014;289(47):32481-7.

45. Wegner N, Wait R, Sroka A, et al. Peptidylarginine deiminase from Porphyromonas gingivalis citrullinates human fibrinogen and $\alpha$ enolase: implications for autoimmunity in rheumatoid arthritis. Arthritis Rheum. 2010;62(9):2662-72.

46. Ireland JM, Unanue ER. Autophagy in antigen-presenting cells results in presentation of citrullinated peptides to CD4 T cells. $\mathrm{J}$ Exp Med. 2011;208(13):2625-32.

47. Ireland J, Herzog J, Unanue ER. Cutting edge: unique T cells that recognize citrullinated peptides are a feature of protein immunization. J Immunol. 2006;177(3):1421-5.

48. Lappin DF, Apatzidou D, Quirke AM, et al. Influence of periodontal disease, Porphyromonas gingivalis and cigarette smoking on systemic anti-citrullinated peptide antibody titres. J Clin Periodontol. 2013 Oct;40(10):907-15.

49.• de Pablo P, Dietrich T, Chapple IL, et al. The autoantibody repertoire in periodontitis: a role in the induction of autoimmunity to citrullinated proteins in rheumatoid arthritis? Ann Rheum Dis. 2014 Mar;73(3):580-6. This study was the first to show low levels of autoreactivity to non-citrullinated variants of rheumatoid autoantigens in patients with periodontitis.
50. Quirke AM, Lugli EB, Wegner N, et al. Heightened immune response to autocitrullinated Porphyromonas gingivalis peptidylarginine deiminase: a potential mechanism for breaching immunologic tolerance in rheumatoid arthritis. Ann Rheum Dis. 2014 Jan;73 (1):263-9.

51. Kharlamova, N., Sherina N, Quirke AM, et al. Antibodies to Porphyromonas gingivalis as an etiological clue to the development of anti-citrullinated protein antibody positive rheumatoid arthritis [abstract]. Arthritis Rheum 2013;65(Suppl 10). doi:10.1002/art. 2013.65.issue-s10.

52. Suzuki A, Yamada R, Chang X, et al. Functional haplotypes of PADI4, encoding citrullinating enzyme peptidylarginine deiminase 4, are associated with rheumatoid arthritis. Nat Genet. 2003;34(4): 395-402.

53. Harvey GP, Fitzsimmons TR, Dhamarpatni AA, et al. Expression of peptidylarginine deiminase- 2 and -4 , citrullinated proteins and anticitrullinated protein antibodies in human gingiva. J Periodontal Res. 2013;48(2):252-61.

54. Vitkov L, Klappacher M, Hannig M, et al. Neutrophil fate in gingival crevicular fluid. Ultrastruct Pathol. 2010;34(1):25-30.

55. Dwivedi N, Radic M. Citrullination of autoantigens implicates NETosis in the induction of autoimmunity. Ann Rheum Dis. 2014;73(3):483-91.

56. Sur Chowdhury C, Giaglis S, Walker UA, et al. Enhanced neutrophil extracellular trap generation in rheumatoid arthritis: analysis of underlying signal transduction pathways and potential diagnostic utility. Arthritis Res Ther. 2014;16(3):R122.

57. Pratesi F, Dioni I, Tommasi C, et al. Antibodies from patients with rheumatoid arthritis target citrullinated histone 4 contained in neutrophils extracellular traps. Ann Rheum Dis. 2014;73(7): 1414-22.

58. Lundberg K, Kinloch A, Fisher BA, et al. Antibodies to citrullinated alpha-enolase peptide 1 are specific for rheumatoid arthritis and cross-react with bacterial enolase. Arthritis Rheum. 2008;58(10):3009-19.

59. Kinloch AJ, Alzabin S, Brintnell W, et al. Immunization with Porphyromonas gingivalis enolase induces autoimmunity to mammalian $\alpha$-enolase and arthritis in DR4-IE-transgenic mice. Arthritis Rheum. 2011;63(12):3818-23.

60. Quirke, A.-M., Perry E, Cartwright A, et al. Bronchiectasis: a model for chronic bacterial infection inducing autoimmunity in rheumatoid arthritis [abstract no. 433]. 2014 ACR/ARHP Annual Meeting; 14-19 Nov 2014; Boston.

61. Chen HH, Huang N, Chen YM, et al. Association between a history of periodontitis and the risk of rheumatoid arthritis: a nationwide, population-based, case-control study. Ann Rheum Dis. 2013;72(7): 1206-11. 